\section{Community-based recruitment of patients with COPD into clinical research}

\begin{abstract}
Identifying subjects for clinical trials is difficult and the evidence base for recruitment strategies is limited, particularly in the field of COPD. We compared the efficiency and patient characteristics of different community-based recruitment strategies during a non-commercial COPD trial in the UK. Recruiting from general practice COPD registers was less efficient and identified patients with significantly milder disease than recruiting through pulmonary rehabilitation and patient groups. We report our experience and propose that pulmonary rehabilitation and patient groups may represent an enriched pool of COPD patients to recruit into clinical trials.

Trial registration number: EudraCT 2011001063-43
\end{abstract}

\section{INTRODUCTION}

Recruitment of patients into clinical trials is challenging and many publicly funded trials in the UK miss their targets. ${ }^{1}$ Many studies investigating COPD may wish to recruit from patient groups or primary care; however, in contrast to the low rates reported by recent large interventional trials (eg, $7 \%{ }^{2}$ ), evidence suggests that patients on UK general practice (GP) databases do not fulfil diagnostic criteria for COPD when retested. ${ }^{3}$ Optimal strategies for recruiting these patients have not been adequately explored.

\section{METHODS}

\section{Recruitment pathways}

Three approaches were used during recruitment of subjects to a recent noncommercial trial of oral antibiotics in stable COPD.

1. Local GP surgeries wrote to patients on their COPD register, and interested patients replied directly to the study team using preaddressed reply slips.

2. Similarly, local pulmonary rehabilitation (PR) groups wrote to COPD patients on their database.

3. Study team members gave educational talks to local PR and patient support groups, and at the end described current research plans. Interested patients approached the study team directly.

Centres were reimbursed for sending letters according to standard research tariffs.

\section{Screening}

Interested patients were contacted and a screening visit arranged which included a full medical history and postbronchodilator spirometry. Patients were considered eligible if COPD was confirmed with $\mathrm{FEV}_{1}<80 \%$ predicted and $\mathrm{FEV}_{1}$ to $\mathrm{FVC}$ ratio $<0.7$.

\section{Analysis}

The efficiency of identifying eligible patients was assessed by comparing the reply and screening failure rates. External time and financial costs were estimated per eligible patient identified, excluding researcher salaries. Patient characteristics were compared by recruitment source.

\section{RESULTS}

Between January 2012 and May 2013, 37 GP surgeries and four PR groups sent letters to 2300 and 469 patients, respectively. Reply rates were similar from both sources (21\% and 22\%) and $156(7 \%)$ and $37(8 \%)$ of these patients attended screening. The educational talks identified 53 patients, of whom 23 (43\%) were screened. Approaches 1 and 2 had screen failure rates of $35 \%$ and $19 \%$, while approach 3 had the lowest $(13 \%)$. Figure 1 summarises these pathways and gives information on the estimated time and financial commitments per eligible patient identified.

Eligible patients recruited from GP surgeries had significantly milder disease than those from $\mathrm{PR}$ and patient groups (mean (SD) $\mathrm{FEV}_{1} 63 \%$ predicted (20) vs $53 \%(18, \mathrm{p}=0.003), \mathrm{FEV}_{1}$ to $\mathrm{FVC}$ ratio $0.55(0.11)$ vs $0.50 \quad(0.11, p=0.007)$, exacerbations in the previous year $1.7(2.0)$ vs $2.7(3.4, p=0.029))$, as well as lesser smoking history and fewer medication

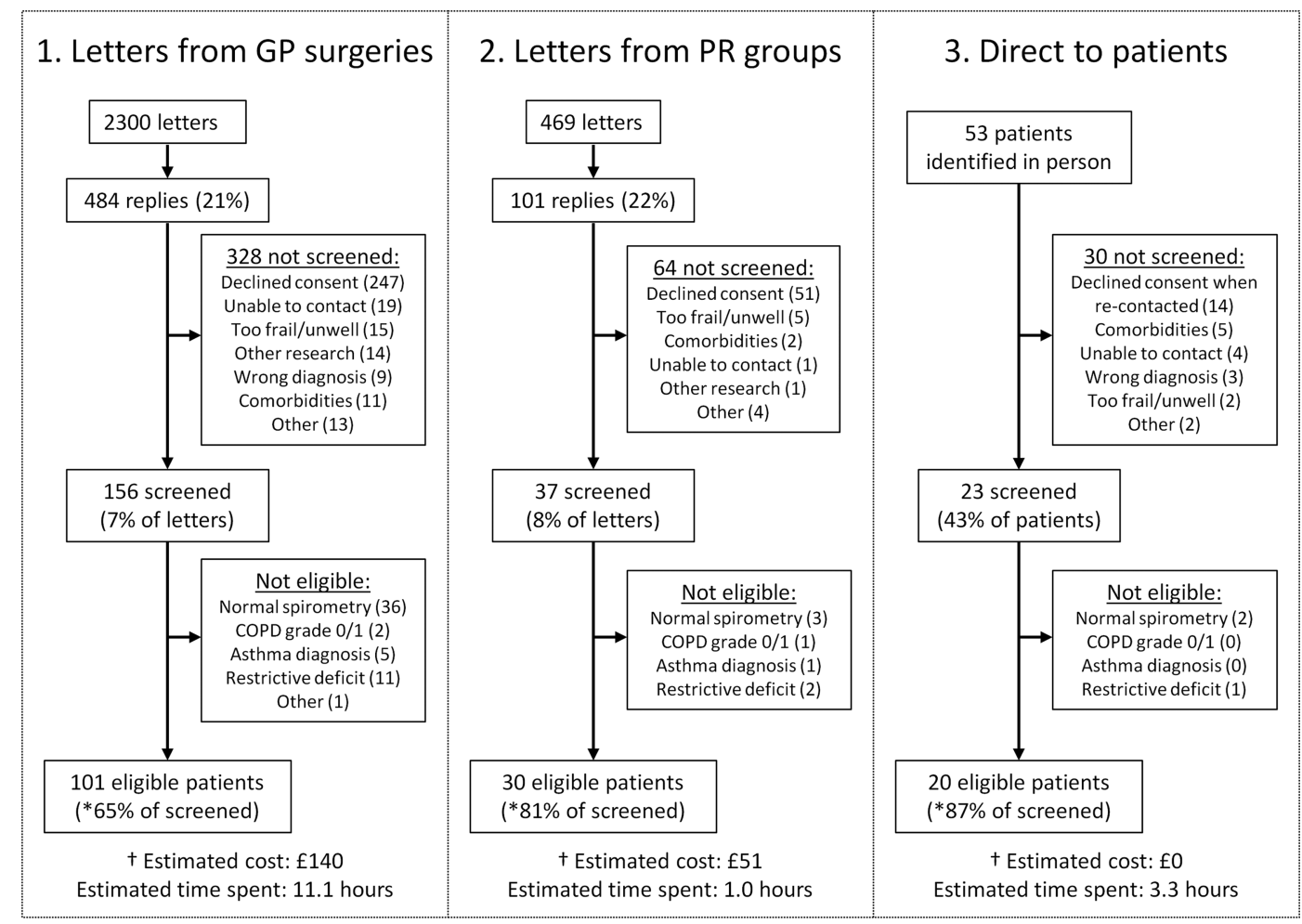

*significant association between referral source and eligibility at screening $\left(\chi^{2}\right.$ test, $\left.p=0.025\right)$

+ time and cost estimates expressed per eligible patient, excluding researcher salaries

Figure 1 Flow diagram detailing the three different recruitment pathways. 
prescriptions. Further information is detailed in the online supplement.

\section{DISCUSSION}

We found that recruiting patients from GP COPD databases was less efficient, more costly, and identified patients with milder disease than through PR and patient groups. However, the pool of patients accessible via GPs was larger and more representative of the wider population. Recruitment via PR and patient groups may therefore target an enriched population useful for smaller studies, and researchers planning future studies of COPD should prioritise resources accordingly.

\section{Simon E Brill, ${ }^{1}$ Ethaar El-Emir, ${ }^{1}$ James P Allinson, ${ }^{1}$ Gavin C Donaldson, ${ }^{1}$ Irwin Nazareth, ${ }^{2}$ Jadwiga A Wedzicha ${ }^{1}$}

${ }^{1}$ Centre for Respiratory Medicine, University College London Medical School, London, UK

${ }^{2}$ Department of Primary Care \& Population Health, University College London Medical School, London, UK

Correspondence to: Dr Simon E Brill, Centre for Respiratory Medicine, University College London Medical School, Royal Free Campus, Rowland Hill Street, London NW3 2PF, UK; simon.brill@nhs.net

Contributors SEB, EE-E, GCD, IN and JAW planned the study and recruitment strategies and wrote the protocols and materials. SEB, EE-E and JPA contacted the individual patient identification centres to organise recruitment. SEB and JPA gave the talks to the patient groups, contacted and screened the patients and collected the data. SEB wrote the first draft and performed the main data analysis. All authors contributed to the data interpretation and to the writing of the paper. All authors approved the final draft. JAW will act as guarantor.

Funding National Institute for Health Research, RP-PG-0109-10056.

Disclaimer This letter presents independent research funded by the National Institute for Health Research (NIHR) under the Programme Grants for Applied Research programme (RP-PG-0109-10056). The views expressed are those of the authors and not necessarily those of the NHS, the NIHR or the Department of Health.

\section{Competing interests None.}

Ethics approval Kings College Hospital Regional Ethics Committee (reference 11/LO/0932)

Provenance and peer review Not commissioned; internally peer reviewed.

Data sharing statement The dataset is available from the corresponding author (simon.brill@ucl.ac.uk).

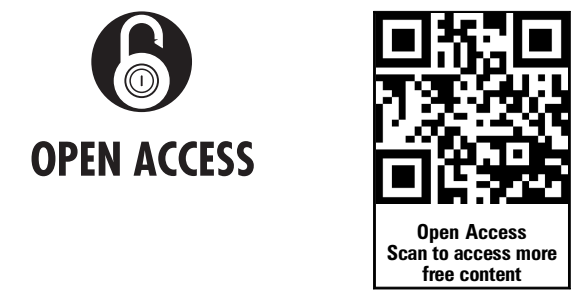

Open Access This is an Open Access article distributed in accordance with the Creative Commons Attribution Non Commercial (CC BY-NC 3.0) license, which permits others to distribute, remix, adapt, build upon this work non-commercially, and license their derivative works on different terms, provided the original work is properly cited and the use is noncommercial. See: http://creativecommons.org/licenses/ by-nc/3.0/

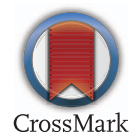

To cite Brill SE, El-Emir E, Allinson J P, et al. Thorax 2014:69:951-952.

Received 7 February 2014

Accepted 12 February 2014

Published Online First 4 March 2014

Thorax 2014:69:951-952.

doi:10.1136/thoraxjnl-2014-205253

- Additional material is published online only. To view please visit the journal online (http://dx.doi.org/10. 1136/thoraxjnl-2014-205253)

\section{REFERENCES}

1 McDonald AM, Knight RC, Campbell MK, et al. What influences recruitment to randomised controlled trials? A review of trials funded by two UK funding agencies. Trials 2006;7:9.

2 Albert RK, Connett J, Bailey WC, et al. Azithromycin for prevention of exacerbations of COPD. $N$ Engl J Med 2011:365:689-98.

3 Jones RC, Dickson-Spillmann M, Mather MJ, et al. Accuracy of diagnostic registers and management of chronic obstructive pulmonary disease: the Devon primary care audit. Respir Res 2008:9:62. 